\title{
SINGLE CHOLINERGIC RECEPTOR CHANNEL CURRENTS IN CULTURED HUMAN MUSCLE
}

\author{
MEYER B. JACKSON, ${ }^{*, 1}$ HAROLD LECAR, ${ }^{*}$ VALERIE ASKANAS, $\neq^{, 2}$ AND W. KING ENGEL ${ }^{2}$ \\ "Laboratory of Biophysics, Intramural Research Program and $\$$ Medical Neurology Branch, National Institutes of \\ Neurological and Communicative Disorders and Stroke, National Institutes of Health, Bethesda, Maryland 20205
}

Received March 3, 1982; Revised May 5, 1982; Accepted May 6, 1982

\begin{abstract}
Single cholinergic channel currents were recorded in adult human muscle tissue culture. The agonists suberyldicholine and carbamylcholine produce channcls with the same conductance as channels produced by acetylcholine but with different closing kinetics. The antagonist tubocurarine, alone or mixed with suberyldicholine, activates channels which close very rapidly. For agonistactivated channels, the distribution of open state lifetimes shows deviations from the usual single exponential form. An excess of short duration openings indicates the presence of an additional faster kinetic process. The lifetime distribution data can be interpreted in terms of varying proportions of slow and fast components which are present in a ratio determined by curve-fitting the appropriate two-exponential function to observed open time distributions. This ratio shows great variability in muscle from older cultures, but the fast and slow time constants are relatively constant. The observation of double exponential open time distributions indicates that the mechanism of channel closing is more complicated than earlier evidence indicated.
\end{abstract}

The prospects for electrophysiological study of adult human muscle have improved considerably as a result of the development of procedures for growing human muscle in tissue culture. Myotubes derived from the satellite cells of human muscle biopsies can be penetrated by microelectrodes; studies of the electrical properties of these cells have been presented (Tahmoush et al., 1980). The acetylcholine sensitivity of tissue- and organ-cultured human muscle has been examined using noise analysis to estimate the basic properties of acetylcholineactivated ionic channels (Bevan et al., 1978; Cull-Candy et al., 1979). Cultured rat and chick myotubes are sensitive to acetylcholine, and direct observation of individual channel currents with an extracellular patch clectrode provides more detail at the molecular level about the nature of this response (Jackson and Lecar, 1979; Nelson and Sachs, 1979). The patch electrode technique for recording single channel currents was developed by Neher and Sakmann (1976) and is applicable to a wide range of systems (Hamill et al., 1981). Applying this technique to adult human muscle culture, we found that the unitary acetylcholine receptor channel properties are similar to those found in cultured rat muscle using the

\footnotetext{
${ }^{1}$ Present address: Department of Biology, University of California, Los Angeles, 405 Hilgard Street, Los Angeles, CA 90024.

${ }^{2}$ Present address: Neuromuscular Center, Department of Neurology, University of Southern California School of Medicine, Los Angeles, CA 90017.
}

same technique (Jackson and Lecar, 1979). In the present studies, we have examined the response of aneurally cultured human muscle to three agonists, acetylcholine, suberyldicholine, and carbamylcholine, during different stages of development. We also have studied the response to tubocurarine, which normally acts as an antagonist but has been shown to have limited agonist-like activity in embryonic cells (Ziskind and Dennis, 1978; Morris et al., 1982). A preliminary account of this work has been presented (Jackson et al., 1980).

Stepwise changes in membrane current indicate the occurrence of opening and closing transitions of ionic channels activated by the binding interaction between agonist and receptor. The kinetic mechanisms of these transitions can be expressed in terms of the probabilities of state lifetimes; thus, single channel data, in the form of lifetime distributions, can be used to explore channel gating in great detail. Recent studies have directed attention to fast openings and closings with the indication that the gating of chemically activated channels is more complex than previously thought (Colquhoun and Sakmann, 1981; Cull-Candy and Parker, 1982; Gration et al., 1982; Jackson and Lecar, 1982; Jackson et al., 1982). In this study, we examine the rapid openings more fully with an emphasis on variability and development.

\section{Materials and Methods}

Tissue cultures of adult human muscle were prepared from muscle biopsies according to previously described 
techniques (Askanas and Engel, 1975). The cultures used in the present studies were derived from muscle biopsies of patients who, after all diagnostic studies were performed, were found not to have a neuromuscular disease. Living cultures were examined every day under phase contrast microscopy. Fusion of myoblasts took place 10 to 14 days after initiation of the cultures, and after 21 days, many long and rounded muscle fibers with bright halos in phase contrast microscopy were observed. Since such fibers are very suitable for microelectrode studies (Tahmoush et al., 1980), cultures between 21 and 48 days of age, containing numerous fibers judged to be optimal from their appearance, were used for the recording of single cholinergic receptor channel currents.

Cultures were transferred to HEPES-buffered Earle's saline for electrophysiological study. Single channel current recordings were made at room temperature (21 to $23^{\circ} \mathrm{C}$ ) according to established procedures (Neher and Sakmann, 1976; Sigworth and Neher, 1980; Hamill et al., 1981). Membrane potential was recorded with a microelectrode filled with $4 \mathrm{~m}$ potassium acetate. The membrane potential was varied by current injection using a second intracellular microelectrode. All recordings were made with holding potentials between -90 and $-100 \mathrm{mV}$. Two- to 5-megohm patch electrodes were filled with dilute solutions of various agonists in the same saline used to bath the cells. Patch electrodes were pressed against the muscle cell surface to form seals with resistances of 30 to 50 megohms. 'Ihe 4- to 5-pA channel current pulses were easily seen under these conditions, although the signal-to-noise ratio was improved further on occasion by the application of gentle suction through the electrode holder to increase the seal resistance to values in excess of 1000 megohms (Sigworth and Neher, 1980).

Channel currents and lifetimes were analyzed individually with the aid of a computer program which scanned digitized records for discontinuities. Events were displayed and examined visually, with pointers indicating the beginning and end of the event as well as the base line and average current while open. The duration and amplitude of each event were computed and stored in a file for further statistical analysis.

A major consideration in the work to be reported is the limitation imposed by the bandwidth of the recording system. With a bandwidth of $1 \mathrm{kHz}$, the rise time for the observed pulses is $160 \mu \mathrm{sec}$. For pulses of less than 700 $\mu \mathrm{sec}$ duration, there is a significant loss of detection efficiency and consequent uncertainty in determining fast kinetic components. Figure $1 C$ shows typical fast channel openings. The figure illustrates the limiting resolution in our duration measurements.

\section{Results}

Agonist-activated channels. Suberyldicholine and acetylcholine ( 0.1 to $0.2 \mu \mathrm{M}$ solutions) and carbamylcholine ( $0.5 \mu \mathrm{M}$ solutions) usually produced channel currents at a frequency ideal for analysis, such that the overlap of
A
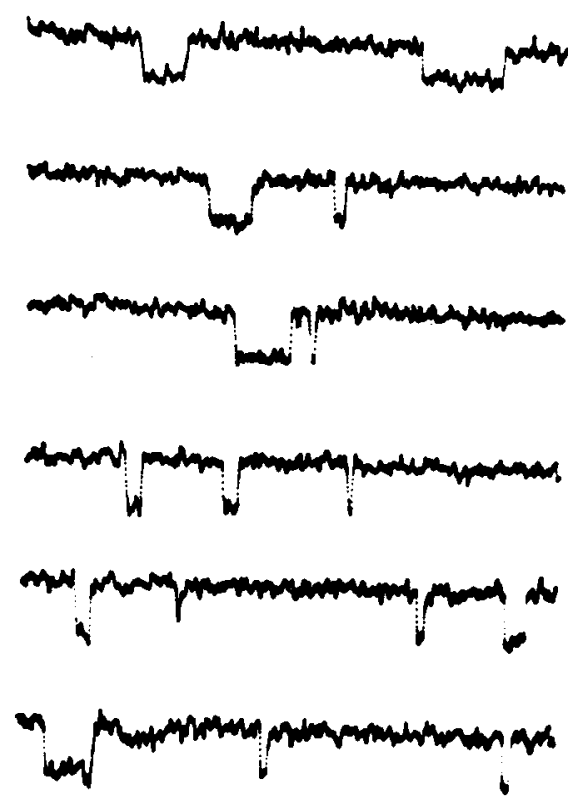

B
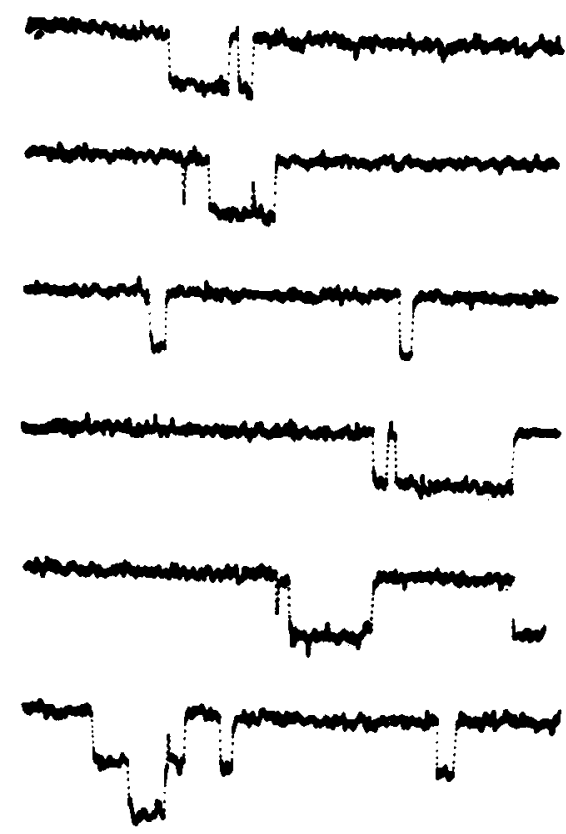

C
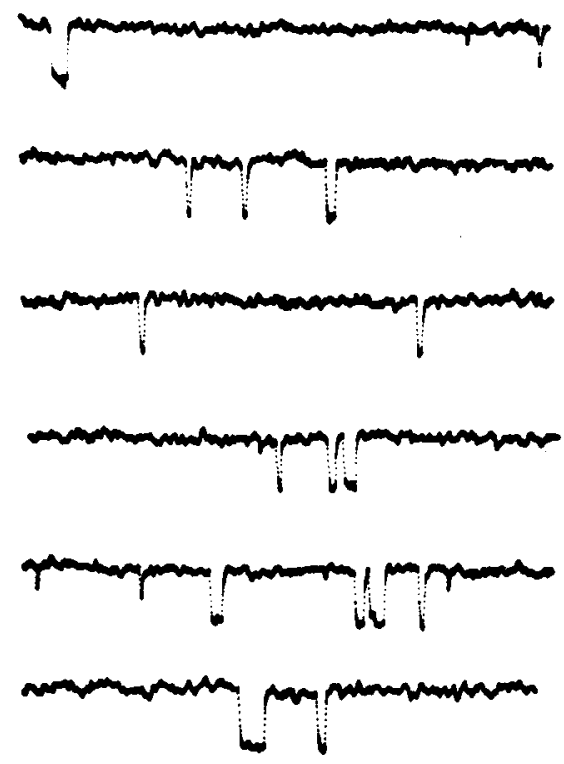

Figure 1. Cultured muscle was bathed in HEPES-buffered Earle's saline at 21 to $23^{\circ} \mathrm{C}$. Myotubes were impaled with two intracellular electrodes and hyperpolarized to -90 and $-100 \mathrm{mV}$. Two- to 5 -megohm patch electrodes were filled with saline containing (A) $0.1 \mu \mathrm{M}$ acetylcholine, $(B) 0.1 \mu \mathrm{M}$ suberyldicholine, or (C) $0.5 \mu \mathrm{M}$ carbamylcholine. The patch electrodes typically formed 30- to 50-megohm seals $(A)$, but occasionally, the seal resistance jumped to values in excess of 1000 megohms $(B$ and $C$ ). Vertical scale bar, $20 \mathrm{pA}$; horizontal scale bar, $205 \mathrm{msec}$. The first trace of $C$ has a $6.4-$ and a 1.1 -msec event. The next trace has events of 1.5, 2.1, and $3.7 \mathrm{msec}$. The first two events of the fifth trace of $C$ are visibly filtered and are only 0.6 and $0.8 \mathrm{msec}$. Thus, we see how the instrumental response time limits the efficiency of detection as the events are filtered down to the noise level. 
TABLE I

Channel conductances and time constants for the three agonists

Channel conductance and fast $\left(\tau_{f}\right)$ and slow $\left(\tau_{s}\right)$ time constants were determined as described in the text. The standard deviations are included and the number of measurements is given in parentheses. It should be noted that the number of measurements of different parameters for the same agonist are not the same. This is because, in some experiments, it was only possible to observe the slow or fast component.

\begin{tabular}{lrrrrrrr}
\hline & \multicolumn{3}{c}{$\begin{array}{c}\text { Channel } \\
\text { Conductance }\end{array}$} & \multicolumn{1}{c}{$\tau_{f}$} & \multicolumn{2}{c}{$\tau_{s}$} \\
\hline & \multicolumn{4}{c}{$p S$} & \multicolumn{4}{c}{ msec } \\
Acetylcholine & $44.7 \pm 2.4$ & $(6)$ & $0.7 \pm 0.2$ & $(4)$ & $4.5 \pm 1.2$ & $(2)$ \\
Suberyldicholine & $43.5 \pm 6.5$ & $(13)$ & $0.8 \pm 0.3$ & $(12)$ & $9.3 \pm 2.4$ & $(13)$ \\
Carbamylcholine & $42.8 \pm 3.7$ & $(2)$ & 0.4 & & $(1)$ & $1.5 \pm 0.1$ & $(2)$ \\
\hline
\end{tabular}

current from different channels was minimal. The relatively uniform frequency of channel events in human muscle cultures can be contrasted with rat muscle cultures where 0.02 to $1.0 \mu \mathrm{m}$ solutions of suberyldicholine often produced channel events of acceptable frequency for analysis (M. B. Jackson, C. E. Morris, B. S. Wong, and $\mathrm{H}$. Lecar, unpublished observation) and large variations in event frequency were encountered for a given concentration of agonist (Jackson et al., 1979). These kinds of interspecific variations were established by using $\alpha$-bungarotoxin-labeling techniques to localize the receptor visually (Askanas et al., 1977). In contrast to rat and chick muscle, the distribution of $\alpha$-bungarotoxin stain in human muscle was thinner and very uniform without foci of increased staining.

Carbamylcholine and suberyldicholine both mimic the action of the transmitter acetylcholine. Sample current records are shown in Figure 1. The rectangular downward pulses are approximately 4 to $5 \mathrm{pA}$ in amplitude. The distributions of amplitudes typically have standard deviations of about $0.5 \mathrm{pA}$. The channel conductance is obtained by averaging many single channel currents and dividing by the holding potential which was always between -90 and $-100 \mathrm{mV}$. The reversal potential was assumed to be $0 \mathrm{mV}$. The conductances obtained for the three different agonists are not significantly different from each other (Table I).

The kinetics of channel closing can be characterized by studying the distribution of channel open times. For the simplest picture of a channel that closes at random with no memory of its initial opening, one would expect the open state lifetimes to be distributed exponentially. Consequently, it is convenient to plot the histogram of durations semilogarithmically. Figure 2 shows semilog plots of the density of events with durations within a given time interval centered at time $t$ and plotted as a function of $t$. From plots such as those of Figure 2, it can be seen that, although the kinetics of closing is not simple, on the average, open times vary in the decreasing order: suberyldicholine, acetylcholine, and carbamylcholine.

One new aspect of channel behavior observed in these experiments is a departure from a single exponential distribution at short times. Thus, in Figure $2, a$ and $b$, there is a considerable excess of channels with durations of less than $2 \mathrm{msec}$, as if there is a second faster component in the channel open time distribution. The appear-

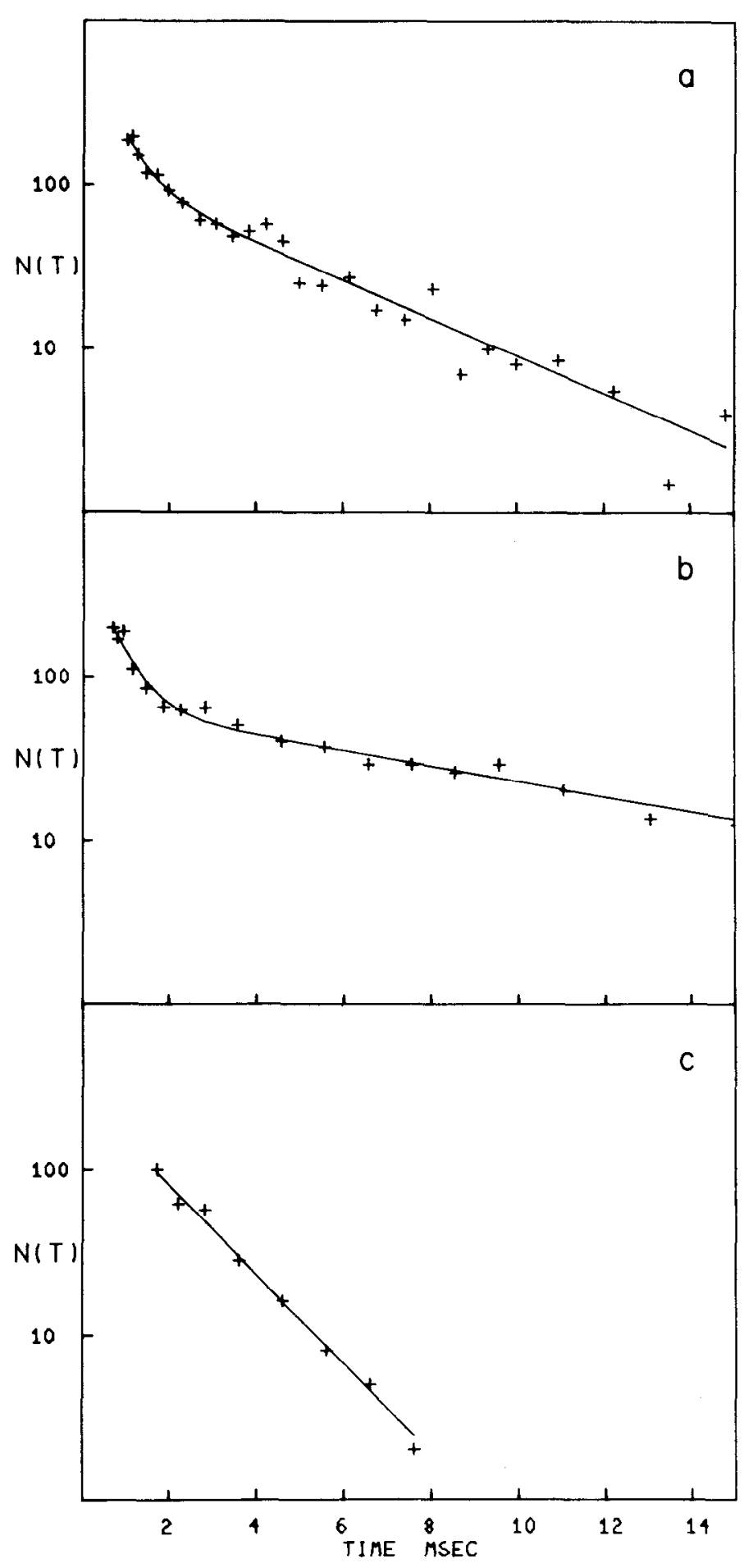

Figure 2. Open state lifetime probability densities for channels activated by $(a)$ acetylcholine $(b)$ suberyldicholine, and $(c)$ carbamylcholine. The solid curves of $a$ and $b$ are best fitting sums of two exponential functions. The solid curve of $c$ is a single exponential function. To obtain these plots, histograms were made with a variable bin width, where the number of events in larger or smaller intervals $(N(T))$ was normalized to give the number of events per msec, and the resultant point was placed at the center of the time interval. The theoretical curves were obtained by least square fits to the points. 
ance of the faster component is variable, but most of the observed distributions are not well fit by a single exponential function. When curve-fitting to a single exponential function, two parameters, the mean open time and the number of channels with zero duration, are varied independently. When a sum of two exponential functions is used and four parameters are varied, a significant improvement is observed in the quality of the fit. The solid curves in Figure 2, $a$ and $b$, are the best fitting sums of the two exponential functions. The distinct upward swing in these distributions and their apparent two-exponential shape suggest a new form of complexity in the kinetics of channel closing. Such deviations from simple first order kinetics have now been reported in several preparations (Rang, 1981; Dionne and Parsons, 1981;
Colquhoun and Sakmann, 1981; Cull-Candy and Parker, 1982; Gration et al., 1982; Jackson and Lecar, 1982; Jackson et al., 1982).

Experiments in which more than about 300 channel events were recorded were analyzed by curve-fitting a sum of two exponential functions to the open time distributions. The average values of these parameters for each agonist are presented in Table I. Even in the best experiments, bandwidth limitations and noise make it difficult to estimate the frequency of channel events reliably when open state lifetimes are less than about 0.6 msec. As a result, the fast component of the distribution is sometimes difficult or impossible to see with the present detection system. Agonists which produce longer mean open times allow an easicr resolution of the fast
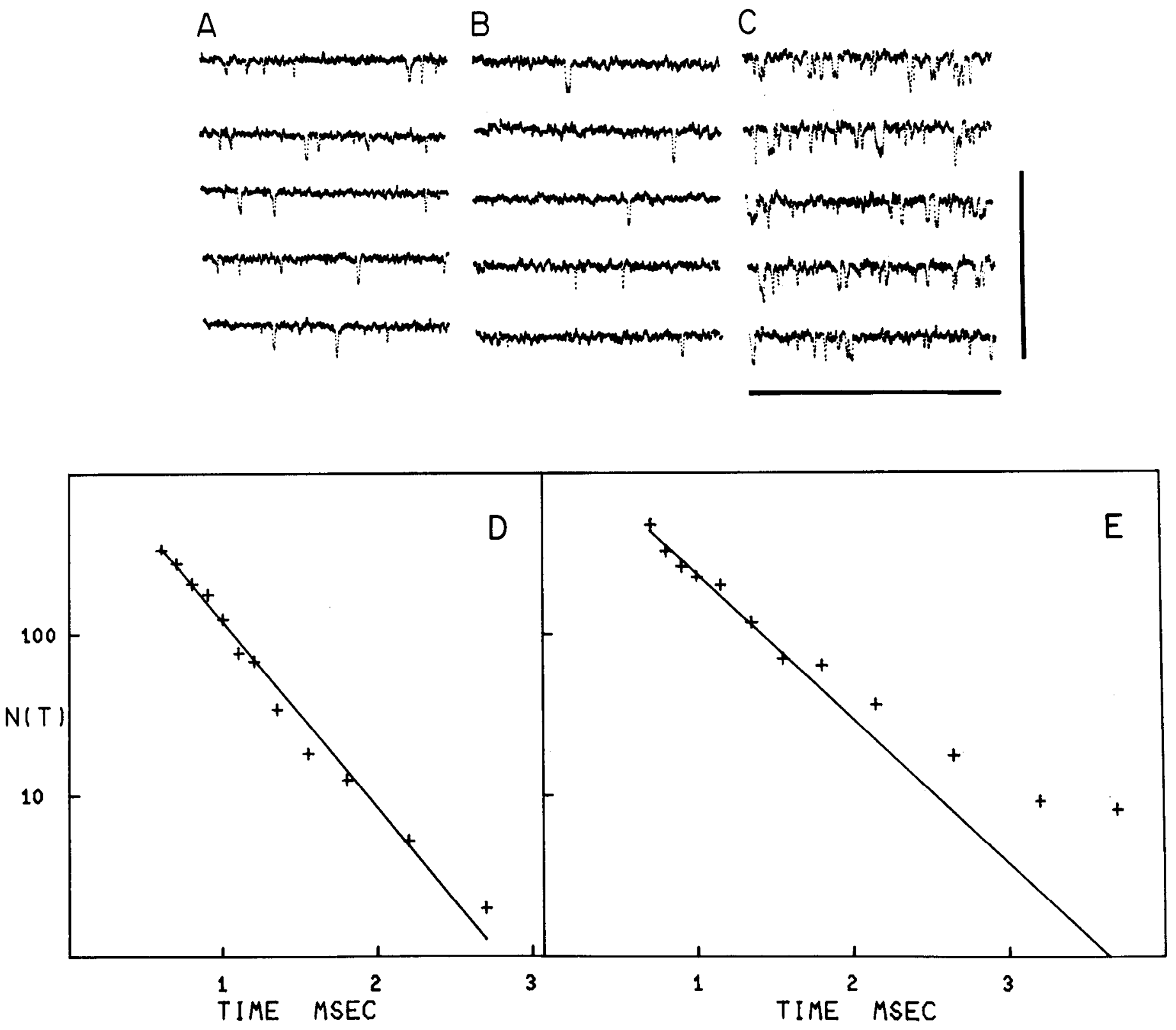

Figure 3. $A$, Channels activated by $10 \mu \mathrm{M}$ tubocurarine; $B$, channels activated by $1 \mu \mathrm{M}$ tubocurarine; $C$, channels activated by a mixture of $1 \mu \mathrm{M}$ tubocurarine and $0.2 \mu \mathrm{M}$ suberyldicholine. Vertical scale bar, $20 \mathrm{pA}$; horizontal scale bar, $102 \mathrm{msec}$. Also shown are open-state lifetime distributions for channels activated by $(D)$ tubocurarine alone and $(E)$ tubocurarine and suberyldicholine. 
component. Hence, the fast component is easily seen with suberyldicholine and acetylcholine but is difficult to see with carbamylcholine.

In order to observe a large fraction of the events, the response time of the recording system must be shorter than the relaxation time of the process that generates the transitions. With suberyldicholine, the short events, which constitute the fast component, had a mean open time (as determined from two-exponential function curve fits) of $0.8 \mathrm{msec}$ (Table I). This value is longer than our recording response time. Faster processes analyzed in this study approach the recording response time, but since, with exponential distributions, there are events of longer duration than the mean, it is still possible for us to infer the presence of a fast process. The critical test is that the recording system cutoff time must be shorter than the longest time at which the fast process appears in the distribution. The clear upward swing in nearly all of the suberyldicholine channel open time distributions is usually evident at times of the order of $2 \mathrm{msec}$ or more and suggests the presence of a distinct additional fast process.

Actions of tubocurarine. Although tubocurarine is a classical antagonist of the nicotinic cholinergic response (Jenkinson, 1960), it has been shown to activate cholinergic channels in cultured and in embryonic rat muscle (Ziskind and Dennis, 1978; Morris et al., 1982). We find that tubocurarine also activates acetylcholine channels in cultured adult human muscle. With tubocurarine alone in the patch electrode, very short-lived channel currents are produced (Fig. 3, $A$ and $B$ ). Because of the uniformity of receptor density in this preparation (Askanas et al., 1977), the frequency of events was reasonably constant for a fixed concentration of channel-activating agent. The frequency of tubocurarine-induced events varies with concentration as illustrated in Figure $3, A$ and $B$, where the concentrations were 10 and $1 \mu \mathrm{M}$, respectively. The infrequent tubocurarine-induced events seen at $1 \mu \mathrm{M}$ concentration (Fig. $3 B$ ) are to be contrasted with the results obtained for a mixture of $1 \mu \mathrm{M}$ tubocurarine and $0.2 \mu \mathrm{M}$ suberyldicholine (Fig. $3 \mathrm{C}$ ). The channels produced by the mixture are too frequent to be activated by tubocurarine alone and too fast to be activated by suberyldicholine alone. This implies that one drug modifies the response of the receptor to the other. Figure $3 D$ shows that tubocurarine alone produces a distribution which is exponential and has a time constant of $0.4 \mathrm{msec}$. The mixture (Fig. $3 E$ ) produces a distribution dominated by one exponential function but with a very small excess of longer duration events which cause the distribution to deviate noticeably from a single exponential function. There were so few events at longer times that a sum of two exponential functions did not improve the quality of the fit, nor did it change the time constant of the fast component from a value of $0.6 \mathrm{msec}$.

It is difficult to determine the conductance when the openings are so fast. The values obtained from these data indicate that the conductance approaches the conductance of agonist-activated channels. Better data from rat muscle gives a conductance for tubocurarine-activated channels which is within a few percent of the conductance values obtained with agonists (Morris et al., 1982).
Tubocurarine is reported to have two actions at the neuromuscular junction, one of which is an interaction with the open state of the channel (Colquhoun et al., 1979; Manalis, 1977). The observation that the channel currents produced by the suberyldicholine/tubocurarine mixture have shorter lifetimes is consistent with tubocurarine blocking the channel after it opens. However, another possibility is that two agonist molecules binding to separate sites cause open states to have different lifetimes, depending upon which sites are occupied. Thus, a mixture of curare and suberyldicholine may have at least three separate lifetimes corresponding to the binding of two tubocararine molecules, two suberyldicholine molecules, or the mixed state. A more thorough study should permit a rigorous discrimination between different models as was accomplished with local anesthetics (Neher and Steinbach, 1978).

The variability of channel properties. The upward swing, or fast component, in the distribution of suberyldicholine channel open times was seen frequently, but there was still a great deal of variability as is illustrated in Figure 4. The data are analyzed to give the number of events which have open times within an interval normalized to $1 \mathrm{msec}$. This is closely proportional to the integral of the probability density function over the time interval taken and can be used as an experimental estimate of probability density. Thus, the data are fit to the function

$$
N(t)=N_{s} e^{-t / \tau_{n}}+N_{f} e^{-t / \tau_{f}}
$$

where $\tau_{f}$ and $\tau_{s}$ are the time constants for the fast and slow components, respectively, and $N_{s}$ and $N_{f}$ are the amplitudes of the intercepts of the individual curves with the zero time axis. $N_{f}$ and $N_{s}$ thus are interpreted as the extrapolated $(e)$ numbers of events with zero duration. The relative areas, $n_{s}$ and $n_{f}$, under the two exponential functions of this empirical density function are obtained by integrating equation 1 to yield the expressions

$$
n_{s}=N_{s} \tau_{s} \quad \text { and } \quad n_{f}=N_{f} \tau_{f}
$$

In order to develop a kinetic interpretation of equation 1 , data from different membrane patches were examined to see how $\tau_{s}$ and $\tau_{f}$ vary from patch to patch. The general trend of the data suggests that the time constant values are relatively invariant but that the relative proportion of the two components varies from patch to patch. Thus, we can use parameters from the two-time constant fit to summarize the variability in terms of the ratio

$$
R=n_{s} /\left(n_{s}+n_{f}\right)
$$

This quantity is the fraction of all events that are slow. Exemplary results are shown in Figure 4 and the parameters derived from the fits are summarized in Figure 5. If the distribution has no upswing but is linear with a slope of $-1 / \tau_{s}$, then $\mathrm{R}$ is 1 . This is nearly the case with Figure $4 a$. On the other hand, with $R=0$, there should be one exponential function with a slope of $-1 / \tau_{f}$. This is illustrated in Figure 4c. The cases like Figure 4, $a$ and $c$, were exceptional, as they are rare examples of distributions where using a sum of two exponential functions did not improve the quality of the fit to the data. In most experiments, results like those in Figures $4 b$ and 2, $a$ and $b$, were obtained with intermediate values of $R$. 
Since $R$ assumes such a wide range of values, systematic variations in other variables should be sought in relation to $R$. In Figure 5, scatter in the experimental values of $\tau_{f}, \tau_{s}$, and $\gamma$ (conductance) is independent of $R$. The range of values of $\tau_{f}, \tau_{s}$, and $\gamma$ is likely to represent normal experimental variation around unique values, whereas variations in $R$ indicate that this quantity is not

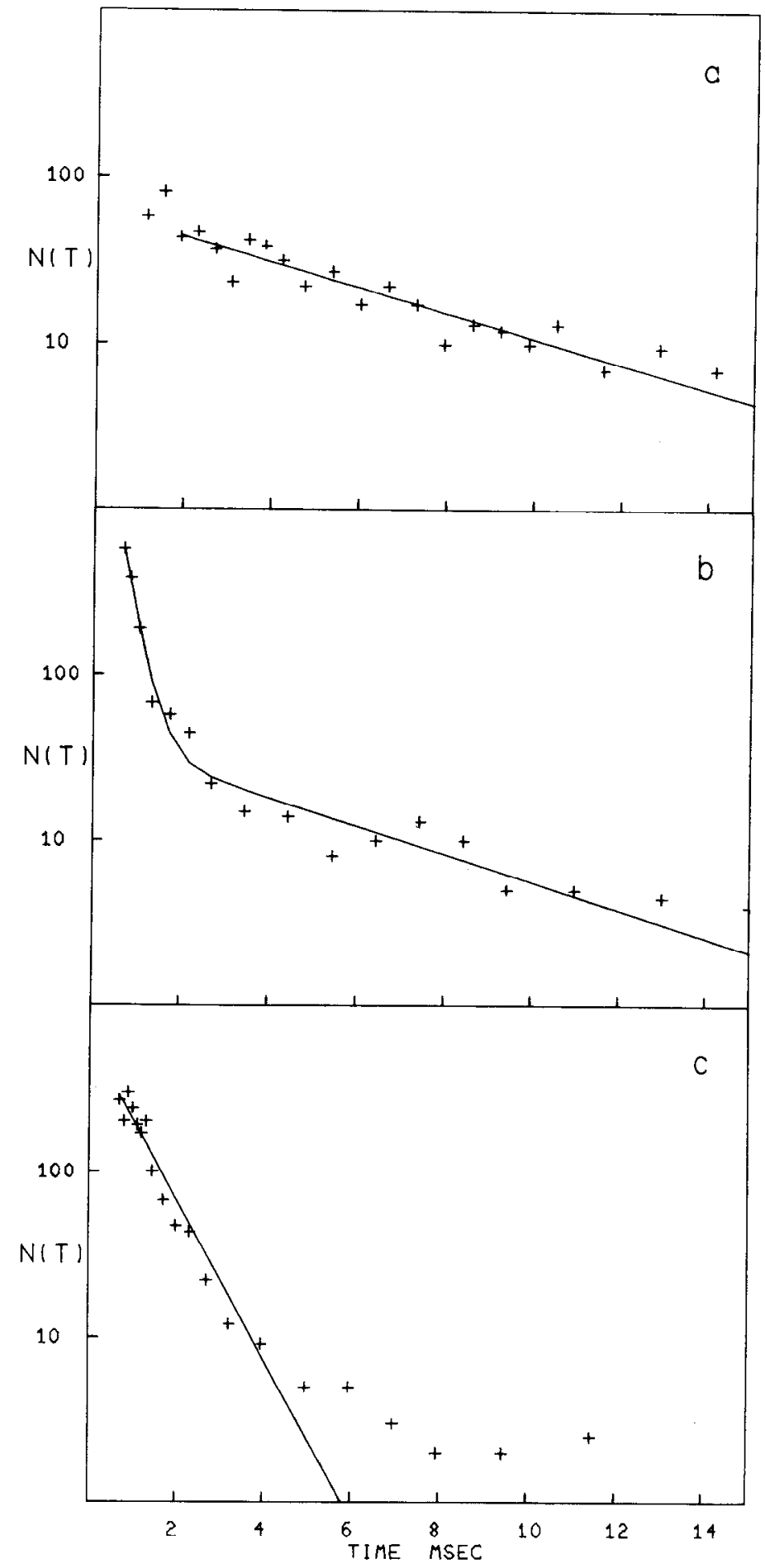

Figure 4. Channel open state lifetime probability densities for three different experiments with suberyldicholine illustrating the variability of $R$, where $R=n_{s} /\left(n_{s}+n_{f}\right)$. Only $b$ was fit by a sum of two exponential functions. The curve fit was done in the same way as for Figure 2.

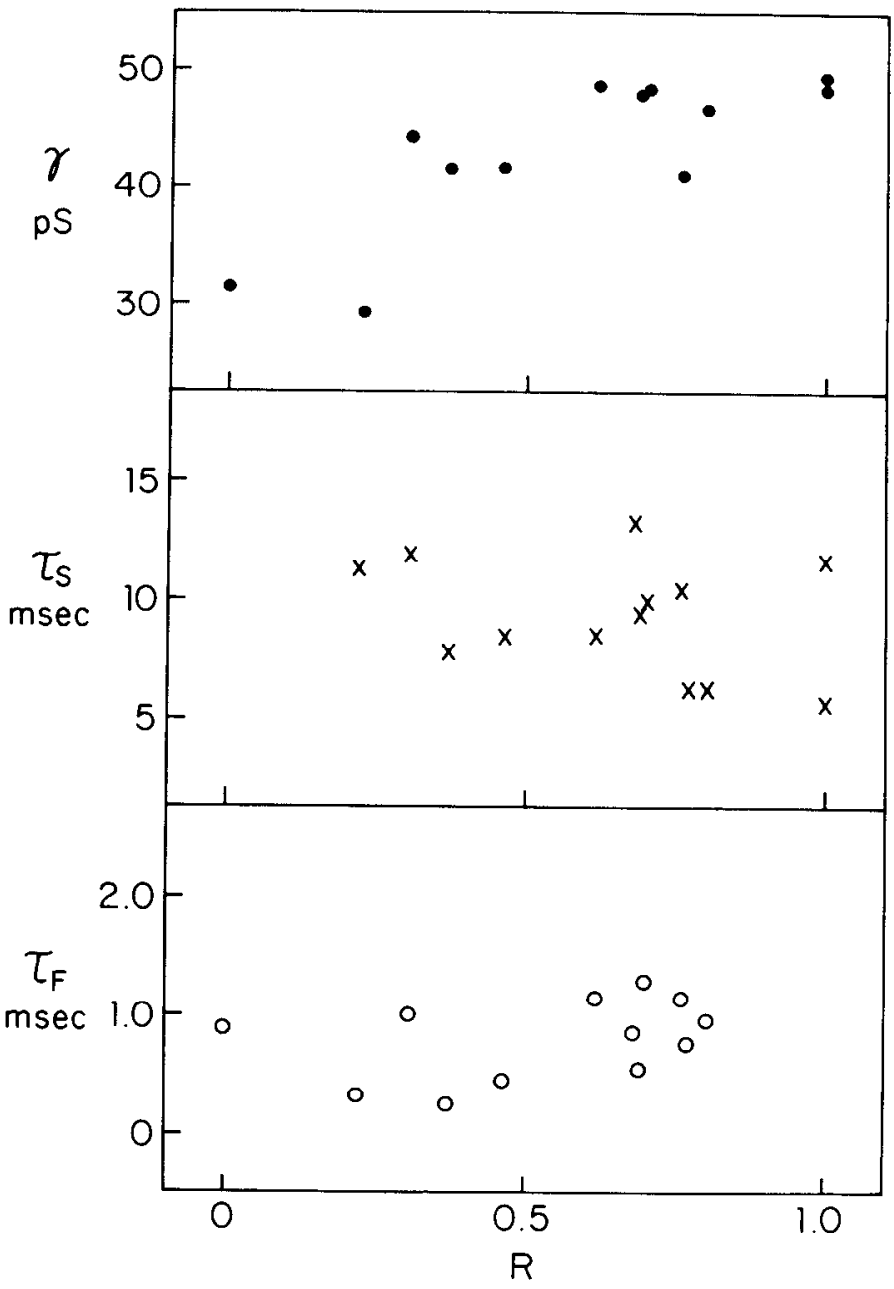

Figure 5. Plots of the channel conductance $(\gamma)$, and $\tau_{s}$, and $\tau_{f}$ versus $R=n_{s} /\left(n_{s}+n_{f}\right)$. Each point represents an experiment on a separate culture.

unique but varies from cell to cell and even from patch to patch in one cell.

\section{Discussion}

In these experiments, we were able to observe agonistinduced current fluctuations caused by single ionic channels. From the single channel currents, it is possible to obtain precise values of the unit conductance and of various kinetic parameters of the gating transitions, such as the closing rates of the open state in the presence of different agonists. The conductance obtained in this study shows nearly quantitative similarity with the value obtained from single channel measurements of embryonic rat muscle (Jackson and Lecar, 1979; Horn and Patlak, 1980). Qualitative similarities in channel closing kinetics are also evident (Jackson and Lecar, 1982; M. B. Jackson, B. S. Wong, C. E. Morris, H. Lecar, and C. N. Christian, submitted for publication).

In most of our experiments, a short duration component appears in the channel open time distribution. Such short duration openings would not contribute appreciably to channel-induced noise as is exemplified in the spectra of earlier noise studies (Bevan et al., 1978; CullCandy et al., 1979). The same amount of power generated 
by the fast process is spread over a much broader range of frequency, with a power per unit of bandwidth which is then too small in amplitude to detect. For example, at low frequency, channels with a 1-msec mean open time generate $1 \%$ of the power per unit of bandwidth that the same number of channels would generate if they were opening with the same rate but with a $10-\mathrm{msec}$ mean open time (Jackson et al., 1982).

The appearance of two kinetic components has been noted in open time distributions obtained from single channel studies in several other preparations. This phenomenon has been observed in frog muscle (Colquhoun and Sakmann, 1981) and in cultured rat muscle (Jackson and Lecar, 1982; M. B. Jackson, B. S. Wong, C. E. Morris, H. Lecar, and C. N. Christian, submitted for publication). GABA-activated channels in spinal cord show a similar effect (Jackson et al., 1982). Two different laboratories report different findings on glutamate-activated channels in locust muscle. Cull-Candy and Parker (1982) see an excess of fast events and Gration et al. (1982) find them to be anomalously scarce. These various observations all have been made very recently, and with improved techniques for single channel recording (Hamill et al., 1981), one may expect significant advances in the study of all aspects of channel-gating kinetics.

We have explored the possibility that the open state duration data can be fit by a sum of two exponential functions. This hypothesis leads to a picture of variability in which the ratio of the two components appears to vary more from patch to patch than the best fit time constants themselves. The analysis of the variable channel kinetics in terms of the relative density of two kinetic components was motivated by the reports of two distinct populations of icetylcholine receptor channels (Fambrough, 1979). Mure rapidly closing channels with higher conductances are seen at endplates, and more slowly closing channels with lower conductances are found in other regions of the muscle. The difference between the values of $\tau_{f}$ and $\tau_{s}$ measured here is too large to correspond to the values for the junctional and extrajunctional receptors reported in the literature. The conductances of the two types of channels differ by 20 to $50 \%$ in the various reports. We could not detect any significant variation in channel conductance that correlates with open state lifetime. In only one experiment was the average current of the faster channels larger than the average current of the slower channels. In all experiments, the channel current was within $0.3 \mathrm{pA}$ of the mean for all values of open state lifetime (provided that the duration is long enough so that current is unaffected by bandwidth). This result is illustrated in Figure 5 where $\gamma$ is plotted versus $R$. The lower values of $\gamma$ for two experiments with $R$ near 0 are a consequence of the difficulty of measuring the conductance of fast channels. Within the limits set by the intrinsic experimental variability, none of these quantities undergoes any significant variation in concert with the variation in $R$.

A hypothesis of two populations of channels is by no means the only possible interpretation of such variable kinetic behavior. Even a single population of channels can obey some non-first order transition scheme and produce open time distributions which are a sum of two exponential functions (Colquhoun and Hawkes, 1981). One example of a complex process which would explain the two-time constant data as well as the tubocurarine/ suberyldicholine interactions is the notion that channels can be opened by the binding of one or two agonist molecules to the receptor and that these two distinct open states have different lifetimes (Colquhoun and Sakmann, 1981; Cull-Candy and Parker, 1982). The alternative that the two processes also could reflect the distribution of one kind of receptor between two very slowly interconverting activable states is still another possibility which is difficult to distinguish from the two populations hypothesis both in principle and in practice. When the receptor density is low, as is the case with locust muscle, and recordings are made from a patch of membrane containing only one channel, the two populations hypothesis can be eliminated (Cull-Candy and Parker, 1982).

The cultures can be maintained for several weeks with morphological changes occurring during that time (Askanas and Engel, 1975). Of the kinetic parameters, $R$ is the quantity which shows the greatest variability; hence, we tested for systemic variation of this parameter with the age of the culture. The solid circles of Figure 6 show the results. If there is a trend evident, five younger cultures ( 3 to 4 weeks) had $R$ values clustered at about 0.7 . Older cultures show no trend at all but are spread over the full range of possible values of $R$. The crosses in Figure 6 show the results of three experiments on one culture which underwent morphological differentiation much earlier than is typical. In all of the other cultures, fusion of satellite cells occurred late in the 2 nd week, but in this culture, fusion occurred after only 1 week. Thus, the spread in $R$ values for this culture correlates with

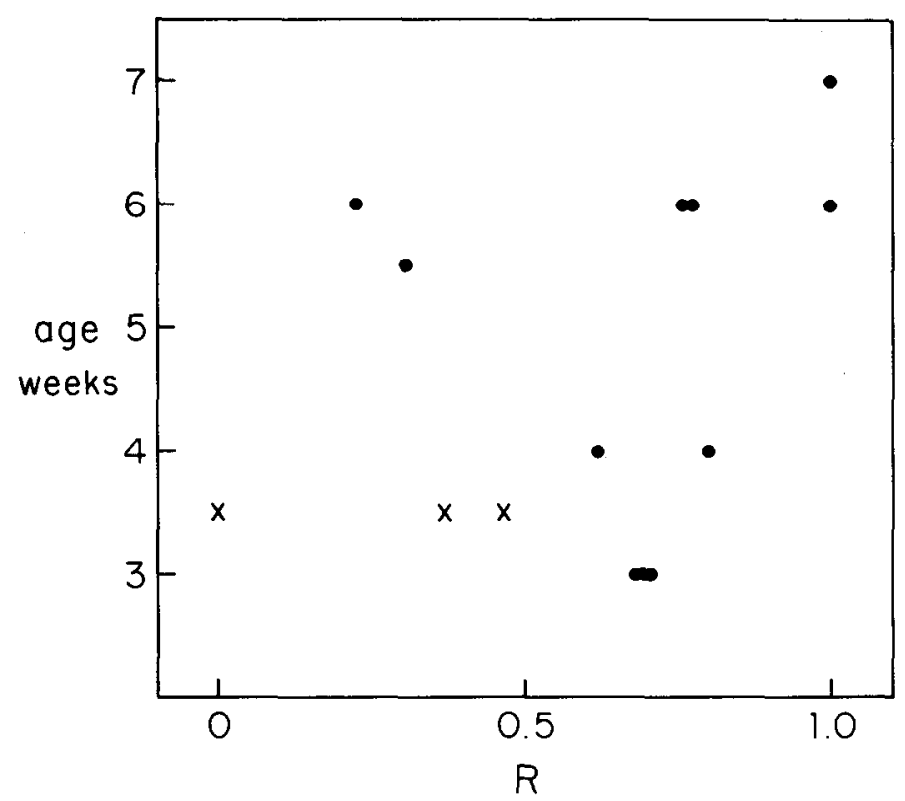

Figure 6. Plot of $R$ versus the age of the cultures. The three points represented by $\times$ are from one culture which showed unusually rapid morphological development, fusing a week earlier than the other cultures. Except for the $\times$ points, each point represents an experiment on a separate culture. 
other indicators of accelerated development but not with the actual age.

Developmental changes in acetylcholine receptor channel properties have been observed in both rat and frog muscle, with channel open state lifetimes decreasing in the course of development (Sakmann and Brenner, 1978; Fischbach and Schuetze, 1980; Kullberg et al., 1981). The results reported here indicate that the channels in human muscle cultures experience a wider variety of change in an undefined direction. With the observation of such a developmental change in vitro, tissue culture offers the unique opportunity of controlling the environment with the hope of determining what conditions specify this direction.

Another potentially interesting indicator of the developmental state of muscle cells is the capacity of channels to be activated by tubocurarine (Ziskind and Dennis, 1978; Morris et al., 1982). It is interesting to find that muscle fibers derived from the satellite cells of adult human muscle have a form of chemosensitivity expressed by rat muscle in an embryonic state of development. The unusual effects of tubocurarine on a rat muscle cell line (L6) may indicate additional developmental diversity (Gartner et al., 1976).

Many studies indicate that the developmental state of muscle has a profound effect on the acetylcholine receptor-channel complex (Sakmann and Brenner, 1978; Fischbach and Schuetze, 1980; Kullberg et al., 1981; Fambrough, 1979). Receptors at the endplate, in denervated supersensitive muscle, and in embryonic muscle exhibit unique properties. The effort made here to identify molecular details with a sensitive channel probe may be of value when attempting to use tissue-cultured muscle to explore these phenomena further. Each aspect of the acetylcholine receptor channel can be studied following a host of developmental manipulations, such as innervation (Fischbach and Cohen, 1973) or treatment with neural factors (Podleski et al., 1978; Christian et al., 1978; Kuromi and Hasegowa, 1975). The present study also offers the prospect of research into the pathology of muscle, since the channel conductance, closing kinetics, pharmacology, and development all can be explored in cultures of muscle from palients wilh different forms of muscular dystrophy and myotonic atrophy, where membrane abnormalities may occur.

\section{References}

Askanas, V., and W. K. Engel (1975) A new program for investigating adult human skeletal muscle grown aneurally in tissue culture. Neurology (N. Y.) 25: 58-67.

Askanas, V., W. K. Engel, S. P. Ringel, and A. N. Bender (1977) Acetylcholine receptors of aneurally cultured human and animal muscle. Neurology (N. Y.) 27: 1019-1022.

Bevan, S., R. W. Kullberg, and J. Rice (1978) Acetylcholineinduced conductance fluctuations in cultured human myotubes. Nature 273: 469-471.

Christian, C. N., M. P. Daniels, H. Sugiyama, Z. Vogel, L. Jacques, and P. G. Nelson (1978) A factor from neurons increases the number of acetylcholine receptor aggregates on cultured muscle cells. Proc. Natl. Acad. Sci. U. S. A. 40114015.

Colquhoun, D., and A. G. Hawkes (1981) On the stochastic properties of single ion channels. Proc. R. Soc. Lond. (Biol.) 211: 205-235.
Colquhoun, D., and B. Sakmann (1981) Fluctuations in the microsecond time range of the current through single acetylcholine receptor ion channels. Nature 294: 464-466.

Colquhoun, D., F. Dryer, and R. E. Sheridan (1979). The actions of tubocurarine at the frog neuromuscular junction. J. Physiol. (Lond.) 293: 247-284.

Cull-Candy, S. G., and I. Parker (1982) Rapid kinetics of single glutamate receptor channels. Nature 295: 410-412.

Cull-Candy, S. G., R. Miledi, and A. Trautmann (1979) Endplate currents and acetylcholine noise at normal and myasthenic human end-plates. J. Physiol. (Lond.) 287: 247-265.

Dionne, V. E., and R. L. Parsons (1981) Characteristics of the acetylcholine operated channel at twitch and slow fibre neuromuscular junctions of the garter snake. J. Physiol. (Lond.) 310: $145-158$.

Fambrough, D. M. (1979) Control of acetylcholine receptors in skeletal muscle. Physiol. Rev. 59: 165-227.

Fischbach, G. D., and S. A. Cohen (1973) The distribution of acetylcholine sensitivity over uninnervated and innervated muscle fibers grown in cell culture. Dev. Biol. 31: 147-162.

Fischbach, G. D., and S. M. Schuetze (1980) A post-natal decrease in acetylcholine channel open time at rat end-plates. J. Physiol. (Lond.) 303: 125-137.

Gartner, T. K., R. Tand, and T. R. Podleski (1976) Genetic and physiological evidence concerning the development of chemically sensitive voltage-dependent ionophores in L6 cells. J. Neurobiol. 7: 537-549.

Gration, K. A. F., J. J. Lambert, R. L. Ramsey, R. P. Rand, and P. N. R. Usherwood (1982) Closure of membrane channels gated by glutamate receptors may be a two step process. Nature 295: 599-601.

Hamill, O. P., A. Marty, E. Neher, B. Sakmann, and F. J. Sigworth (1981) Improved patch-clamp techniques for high resolution current recording from cells and cell-free membrane patches. Pflugers Arch. 391: 85-100.

Horn, R., and J. B. Patlak (1980) Single channel currents from excised patches of muscle membrane. Proc. Natl. Acad. Sci. U. S. A. 77: 6930-6934.

Jackson, M. B., and H. Lecar (1979) Single post-synaptic channel currents in tissue cultured muscle. Nature 282: 864-865.

Jackson, M. B., and H. Lecar (1982) Double-exponential channel current lifetime distributions in cultured nerve and muscle. Biophys. J. 37: 310a.

Jackson, M. B., C. N. Christian, and H. Lecar (1979) Singlechannel currents of acetylcholine receptors in cultured rat muscle. Soc. Neurosci. Abstr. 5: 482.

Jackson, M. B., H. Lecar, V. Askanas, and W. K. Engel (1980) Single acetylcholine channels in cultured human muscle. Soc. Neurosci. Abst. 6: 778.

Jackson, M. B., H. Lecar, D. A. Mathers, and J. L. Barker (1982) Single channel currents activated by $\gamma$-aminobutyric acid, muscimol, and (-)-pentobarbital in cultured mouse spinal neurons. J. Neurosci. 2: 889-894.

Jenkinson, D. J. (1960) The antagonism between tubocurarine and substances which depolarize the motor end-plate. J. Physiol. (Lond.) 152: 309-324.

Kullberg, R. W., P. Brehm, and J. H. Steinbach (1981) Nonjunctional acetylcholine receptor channel open time decreases during development of Xenopus muscle. Nature 289: 411-413.

Kuromi, H., and S. Hasegawa (1975) Neurotrophic effect of spinal cord extract on membrane potentials of organ-cultured mouse skeletal muscle. Brain Res. 100: 178-181.

Manalis, R. S. (1977) Voltage-dependent effects of curare at the frog neuromuscular junction. Nature 267: 266-268.

Morris, C. E., M. B. Jackson, H. Lecar, B. S. Wong, and C. N. Christian (1982) Activation of individual acetylcholine channels by curare in embryonic rat muscles. Biophys. J. 37: 19a. 
Neher, F., and B. Sakmann (1976) Single-channel currents recorded from membrane of denervated frog muscle fibers. Nature 260: 799-802.

Neher, E., and J. H. Steinbach (1978) Local anaesthetics transiently block currents through single acetylcholine-receptor channels. J. Physiol. (Lond.) 277: 153-176.

Nelson, D. J. and F. Sachs (1979) Single ionic channels observed in tissue-cultured muscle. Nature 282: 861-863.

Podleski, T. R., D. Axelrod, P. Raudin, I. Greenberg, M. M. Johnson, and M. M. Salpeter (1978) Nerve extract induces increase and redistribution of acetylcholine receptors on cloned muscle cells. Proc. Natl. Acad. Sci. U. S. A. 75: 20352039.

Rang, H. P. (1981) The characteristics of synaptic currents and responses to acetylcholine of rat submandibular ganglion cells. J. Physiol. (Lond.) 311: 23-55.

Sakmann, B., and H. B. Brenner (1978) Change in synaptic channel gating during neuromuscular development. Nature 276: $401-402$.

Sigworth, F. J., and E. Neher (1980) Single $\mathrm{Na}^{+}$channel currents observed in cultured rat muscle cells. Nature 287: 447449.

Tahmoush, A. J., G. K. Bergey, V. Askanas, P. G. Nelson, and W. K. Engel (1980) Electrophysiologic properties of aneurally cultured muscle from myotonic atrophy (MA) patients compared with controls. Neurology (N. Y.) 30: 404.

Ziskind, L., and J. J. Dennis (1978) Depolarising effect of curare on embryonic rat muscles. Nature 276: 622-623. 